\title{
Inductive detection of magnetic vortex gyration
}

\author{
Hauke H. Langner, ${ }^{1, *}$ Lars Bocklage, ${ }^{2,1}$ Toru Matsuyama, ${ }^{1}$ and Guido Meier ${ }^{1}$ \\ ${ }^{1}$ Institut für Angewandte Physik und Zentrum für Mikrostrukturforschung, Universität Hamburg, Jungiusstraße 11, 20355 Hamburg, Germany \\ ${ }^{2}$ Deutsches Elektronen-Synchrotron, Notkestraße 85, 22607 Hamburg, Germany
}

(Received 28 September 2012; revised manuscript received 5 December 2012; published 25 February 2013)

\begin{abstract}
Vortex cores in Landau-domain patterns are resonantly excitable by alternating magnetic fields in the subgigahertz regime. We present a highly sensitive method to detect the vortex gyration in single micrometer-sized elements spectroscopically by measuring spectra of induction voltages caused by the stray fields of a single ferromagnetic square exposed to an alternating Oersted field. A distinct change of the induction voltage is observed around the resonance frequency of the vortex core. The shape of the measured spectra deviates from Lorentzian profiles due to voltages induced by magnetic fringing fields of the exciting currents. An analytical description of the measured spectra is given. A characteristic frequency shift in external magnetic fields proves that signals detected by the induction sensor originate from the dynamically excited Landau-domain pattern. The measurements on a single square are compared with measurements on an ensemble of uniform squares.
\end{abstract}

DOI: 10.1103/PhysRevB.87.064420

PACS number(s): 75.78.Fg, 75.50.Bb, 75.70.Ak, 75.70.Kw

\section{INTRODUCTION}

Thin soft magnetic film elements develop characteristic magnetization patterns that spark interest in fundamental research. ${ }^{1}$ Especially permalloy $\left(\mathrm{Ni}_{80} \mathrm{Fe}_{20}\right)$ elements are extensively investigated because this material has vanishing crystalline anisotropy; i.e., only the exchange energy and the strayfield energy significantly contribute to the magnetic properties. Transport measurements such as pulsed inductive microwave magnetometry ${ }^{2,3}$ and absorption spectroscopy $y^{4,5}$ allow one to examine the magnetization dynamics of films and ensembles of uniform elements. But uniformity can be a challenge because a single element never equals another one on the scale of single crystallites. Defects and roughnesses can lead to a modified magnetic behavior such as different switching fields ${ }^{6}$ and different resonance frequencies ${ }^{7}$ of magnetic patterns in alternating magnetic fields. The average over many elements might inhomogeneously broaden the signals. Brillouin light scattering spectroscopy allows one to investigate spectra of spin-wave modes in single thin-film elements at frequencies above $2 \mathrm{GHz} .{ }^{8}$ With time-resolved scanning Kerr microscopy also investigations at lower frequencies are possible. ${ }^{8-10}$ The spatial resolution is limited either by the diameter of the laser spot or by the wavelength of the light. X-rays enable spatial resolutions in the regime of nanometers. Via the x-ray magnetic circular dichroism even magnetic vortices can be resolved with time-resolved scanning $\mathrm{x}$-ray microscopy ${ }^{11-13}$ or time-resolved photoemission electron microscopy. ${ }^{14-17}$ The drawback of these two methods is the availability of beam time and the limited precision in frequency. Electrical detection methods on single elements fill the gap between ensemble transport spectroscopy and microscopic measurements on single elements. Nakano et al. detected the vortex gyration in single disks with magnetic tunnel junctions. ${ }^{18}$ We use a highly sensitive inductive technique to study single squares with a Landau-domain pattern spectroscopically. A micrometer-sized induction loop is placed above the element in order to measure the change of the magnetic flux. In contrast to other work that investigated ferromagnetic wires ${ }^{19,20}$ we aim at electrical detection of the dynamics of a single magnetic vortex. ${ }^{21}$ The gyrotropic mode of the vortex core is identified by a characteristic resonance shift in static external fields ${ }^{13}$ where the shape of the resonance curve can be described analytically.

\section{EXPERIMENTAL SETUP}

The change of the magnetic flux $\phi$ that penetrates an induction loop causes a voltage $V_{\text {ind }}$ according to Faraday's law of induction. The magnetic flux is the product of the magnetic induction $\mathbf{B}$ with the area $A$ surrounded by the loop and the unit vector $\boldsymbol{e}_{z}$ perpendicular to the plane loop. Thus the induction voltage is given by

$$
V_{\text {ind }}=-\frac{d\left(A \mathbf{B} \boldsymbol{e}_{z}\right)}{d t} .
$$

In this work the induction voltage is used to detect magnetization dynamics in a micrometer-sized permalloy square that is exposed to an alternating in-plane Oersted field. The vortex performs a gyrotropic motion ${ }^{12,22}$ if the excitation frequency matches its resonance frequency. The magnetic stray field of the square changes periodically with the gyration frequency of the vortex. The induction loop senses the stray-field variation induced by the vortex motion. Only the $z$ components account for the measurable magnetic flux because the loop on top of the square is prepared parallel to the film plane as shown in the inset of Fig. 1. The stray fields of the domains and the domain walls can be detected rather than the stray field of the vortex itself $^{23}$ because of its small extension of a few nanometers. ${ }^{24}$

The detection of the gyration in a square by an induction loop requires the deposition of several materials, laterally defined by electron-beam lithography. First a gold stripline with a thickness of $57 \mathrm{~nm}$ is evaporated on a gallium arsenide wafer with an adhesive chromium layer. The current through the stripline alternates with frequencies in the gigahertz regime and generates the Oersted fields that drive the vortices. To avoid topological and magnetic inhomogeneities in the permalloy due to the surface roughness of the subjacent gold layer, hydrogen silsesquioxane (HSQ), a negative electron-beam resist, is coated on the stripline before the permalloy film is deposited. This assures a plane surface. HSQ is mixed with methylisobutylketone (MIBK) to reduce the layer thickness. With an atomic force microscope a root-mean-square rough- 


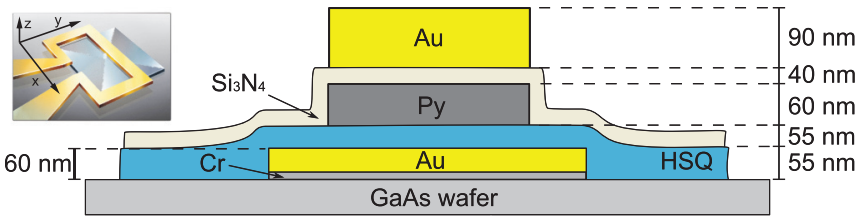

FIG. 1. (Color online) Illustration of the stack of materials. The inset shows a schematic of an induction loop that covers one half of a permalloy square.

ness of the HSQ layer of $0.2 \mathrm{~nm}$ is measured. This roughness is $88 \%$ less than the one of the underlying gold layer. The permalloy square on top of the HSQ layer has a lateral size of $2.2 \mu \mathrm{m}$ and a thickness of $60 \mathrm{~nm}$. Subsequently a $40-\mathrm{nm}$ thick silicon nitride layer is deposited with plasma-enhanced chemical vapor deposition (PECVD). This method provides an isotropic coverage including the edges of the stripline and the permalloy square. Thus the loop, placed on top of the stack of materials, has neither electrical contact to the stripline nor to the permalloy. Since the loop is electrically insulated from the stripline and not sensitive to in-plane field components, only a small fraction of the exciting signal is transmitted into the loop by out-of-plane components of the Oersted field. Thus the layer sequence allows one to resolve small induction voltage signals.

Via simulations the change of the magnetic flux through the induction loop above the excited Landau-domain pattern and the concomitant induction voltage can be determined. Figure 2(a) shows the induction voltages, calculated from simulations with the OOMMF code, ${ }^{25}$ at eight phases of a vortex gyration. Voltages lower than $-660 \mathrm{pV}$ are colored in dark red. The induction loop is located $40 \mathrm{~nm}$ above a permalloy square with a lateral size of $2.2 \mu \mathrm{m}$ and a thickness of $60 \mathrm{~nm}$. The gyromagnetic ratio $\gamma=2.21 \times 10^{5} \mathrm{mC}^{-1}$, the saturation magnetization $M_{\mathrm{S}}=8 \times 10^{5} \mathrm{Am}^{-1}$, a Gilbert damping $\alpha=$ 0.01 , and the exchange constant $A=1.3 \times 10^{-11} \mathrm{Jm}^{-1}$ of

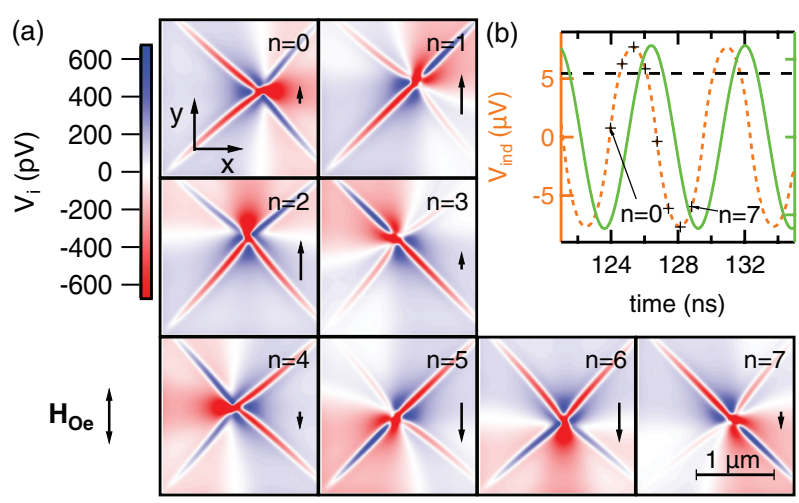

FIG. 2. (Color online) (a) Simulation of the spatially resolved induction voltages $V_{i}$ generated by the flux change $40 \mathrm{~nm}$ above a gyrating Landau-domain pattern. Eight vortex positions with the phases $\varphi=2 \pi n / 8$ are shown. The arrows in each graph indicate the strength and the direction of the external Oersted field. (b) Total induction voltage $V_{\text {ind }}$ versus time for a loop as indicated in the inset of Fig. 1 (orange dotted line) and negative time derivation of the external field in arbitrary units versus time (green solid line). The pluses correspond to the graphs in (a). The dashed line represents the effective value of the induction voltage. permalloy were used. ${ }^{12}$ For the calculation of the induction voltage the area of the permalloy square is discretized. Thus the simulation delivers the induction voltage $V_{\text {ind }}=\sum_{i} V_{i}=$ $\sum_{i} B_{i} \Delta A / \Delta t$, where $i$ numbers the simulation cells that all have the same area of $\Delta A=5 \times 5 \mathrm{~nm}^{2}$. The time difference between the iteration steps is $\Delta t$. To determine the excitation amplitude for the simulations we use values of our experiments. In the experiment the amplitude of the output voltage of the stripline measured with an oscilloscope is $67 \mathrm{mV}$. A loss of $3 \mathrm{~dB}$ from the stripline to the oscilloscope is considered. Thus the alternating Oersted field directly above the stripline has an amplitude of approximately $0.21 \mathrm{mT} .^{2}$ In the simulations the vortex dynamics is simulated by applying a sinusoidal external magnetic field with this amplitude and a frequency of $178 \mathrm{MHz}$ that equals the resonance frequency of the vortex. The steady state of the vortex motion is reached after $100 \mathrm{~ns}$.

The vortex core is the fastest moving part of the Landaudomain pattern so that the maximal flux change occurs in the simulation cell that is closest to the vortex position. Still its contribution to the total flux change is comparatively low. Also the domain walls give rise to strong changes of the flux that weaken from the center to the corners where the domain walls are pinned. The induction voltages of all simulation cells in the induction loop are summed up to obtain the induction voltage $V_{\text {ind }}$. One half of the total flux above the square is always compensating the opposite half. Thus, a symmetric position of the loop would result in an induction voltage close to zero. We calculated several geometries of the loop and found that it is appropriate to cover only one half of the square as illustrated in the inset of Fig. 1 so that the gyrating vortex periodically moves in and out of the area encircled by the loop. For a radius of the vortex trajectory of about $190 \mathrm{~nm}$ an induction signal in the microvolt regime is expected as plotted in Fig. 2(b). With the integrating setup described in the following section we will measure the effective value of the voltage that is $1 / \sqrt{2}$ of the amplitude. The effective voltage is indicated by the black dashed line in Fig. 2(b).

Figure 3 shows a schematic of the setup for the inductive detection. The inset depicts a scanning electron micrograph of the loop prepared above the permalloy square. Magnetization dynamics in the permalloy square is excited by radio-frequency Oersted fields of the signal line. The stripline is matched to

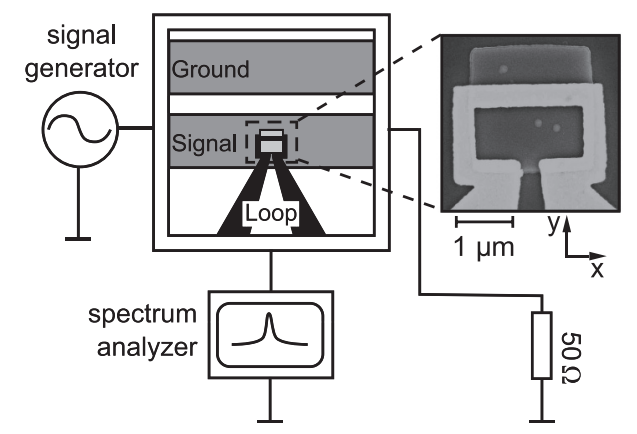

FIG. 3. Schematic of the measurement setup for the inductive detection. The induction loop on top of the permalloy square is positioned above the signal line of a waveguide in ground-signal geometry. The inset shows a scanning electron micrograph of the induction loop above the permalloy square. 
a $50 \Omega$ impedance to avoid wave reflections. The spectrum analyzer connected to the induction loop is set to measure the power at the frequency of the signal generator. The induction voltage is determined from the power by $U=\sqrt{50 \Omega \times P}$. Due to the small induction voltages below microvolts the signal cannot be detected directly. A superimposed signal in the range of $200 \mu \mathrm{V}$ originating from the stripline must be eliminated. To achieve this, first the induction voltage is measured at a specific frequency and external field $H_{\text {ext }}$. This signal contains the information about the magnetization dynamics in the square. Then a reference measurement is performed at the same frequency but with an external field of $50 \mathrm{mT}$ that saturates the magnetization of the permalloy and consequently excludes vortex motion. The difference of the two measurements delivers the differential induction voltage $\Delta V_{\text {ind }}$. So ideally we only obtain a nonzero signal if the magnetization pattern changes. With this setup the noise of the measured voltage has only a root-mean-square value of about $8 \mathrm{nV}$ at $100 \mathrm{MHz}$.

\section{EXPERIMENTAL RESULTS}

It is known that the vortex resonance frequency changes for increasing static external magnetic fields. ${ }^{5,13,26}$ In permalloy squares the resonance shift depends on the direction of the external field. In squares a redshift is expected for fields applied along an edge of the square, a blueshift if the field is applied along a diagonal. ${ }^{13}$ With the precondition that the vortex trajectory should be in the area encircled by the loop for one half of the period the external field has to be applied in the $y$ direction. Then the vortex is only deflected along the $x$ axis that is defined in Fig. 1. Thus a redshift of the resonance frequency is expected. We measure the differential induction voltage $\Delta V_{\text {ind }}$ for external fields $H_{\text {ext }}$ between $-22 \mathrm{mT}$ and $22 \mathrm{mT}$ and frequencies between $50 \mathrm{MHz}$ and $300 \mathrm{MHz}$. The spectra are shown in Fig. 4. For the vortex gyration a resonance frequency of about $178 \mathrm{MHz}$ is predicted in simulations if no external field is applied. This frequency coincides with the measured resonance in the induction spectra if the resonance is interpolated to zero field. The induction signals exhibit a distinct redshift if the static external magnetic field is swept to higher magnitudes. The decrease of the resonance frequency

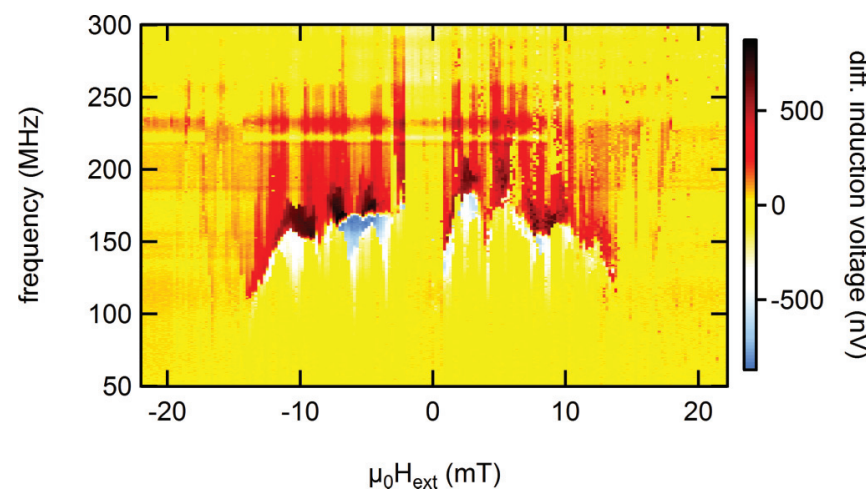

FIG. 4. (Color online) Differential spectra of the induction voltage $\Delta V_{\text {ind }}$ versus external magnetic field for a $2.2 \mu$ m wide and $60 \mathrm{~nm}$ thin permalloy square. The external magnetic field $H_{\text {ext }}$ is applied along the $y$ direction. indicates that the observed magnetic dispersion is caused by a resonantly excited vortex in the permalloy square as it coincides with expectations for vortex motions in nonharmonic potentials. $^{4,13,27}$ From the simulation in Fig. 2 an induction voltage of about $5 \mu \mathrm{V}$ is expected from the gyration. In the measurements we reach a maximum induction voltage of about $1 \mu \mathrm{V}$. The reason for the discrepancy is that the measured induction voltage is a superposition of the voltage induced by the $z$ component of the Oersted field from the stripline and the voltage induced by magnetization dynamics in the permalloy square. In general the two voltages are out of phase (see Sec. IV). Further a signal loss from the induction loop to the spectrum analyzer and the finite loop thickness of about $90 \mathrm{~nm}$ cause deviations from the simulations. Thus the magnitude of the measured voltage is reasonable.

To facilitate the interpretation of the signal obtained from the induction loop we compare the induction voltage spectra with ensemble microwave absorption measurements. Three rows of a total of 1200 permalloy squares with nominally the same size as the single one prepared for the inductive detection are deposited on the substrate. The center-to-center distance between adjacent squares is $3 \mu \mathrm{m}$. A $9-\mu \mathrm{m}$-wide stripline is prepared upon the squares. A part of the sample is shown in Fig. 5(a). Similar to the previously described setup the signal generator supplies the excitation via Oersted fields. The $50 \Omega$ resistor is replaced by the spectrum analyzer maintaining a $50 \Omega$ matching condition to directly measure the absorbed microwave power. Figure 5(b) shows spectra of the vortex resonance of the permalloy squares in the sub-gigahertz regime. Increasing external field strengths shift the frequency slightly. For fields larger than about $7 \mathrm{mT}$ a

(a)

(c) frequency $(\mathrm{MHz})$
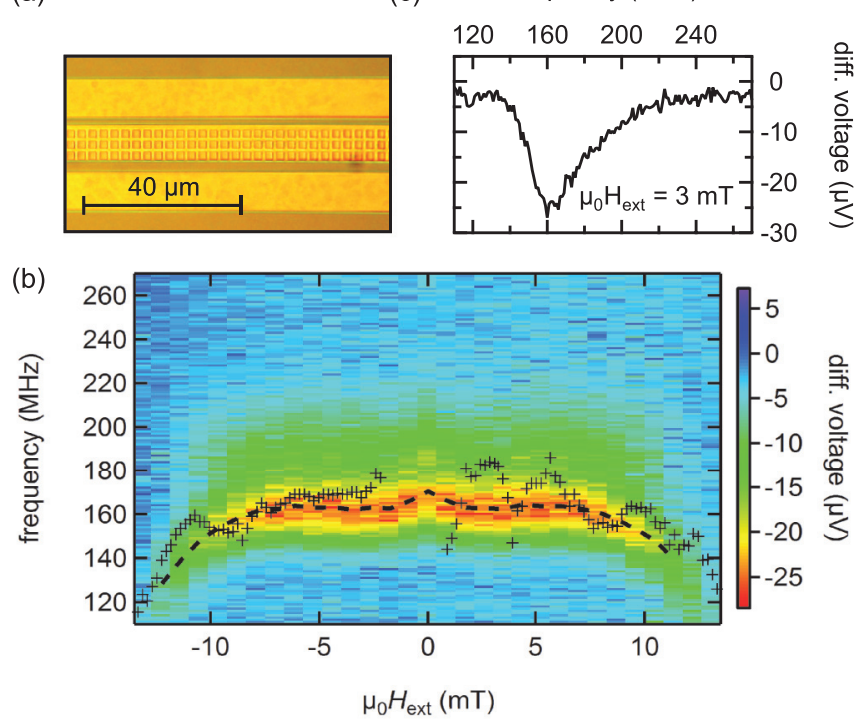

FIG. 5. (Color online) (a) Optical micrograph of an excerpt of the investigated sample with 1200 permalloy squares below a stripline. (b) Absorption spectra of the 1200 permalloy squares in different external magnetic fields. The dashed line corresponds to the absorption maxima determined by Lorentzian fits. The pluses indicate the resonance frequencies obtained from the induction voltage spectra in Fig. 4 from a single square. (c) Absorption spectrum at an external field of $3 \mathrm{mT}$. 
strong redshift occurs that has been discussed above and described elsewhere. ${ }^{13}$ The dashed line represents maxima of Lorentzian fits to the absorption at the accordant external field. While the absorption measurements deliver approximately Lorentz-like resonance curves with a single minimum [see Fig. 5(c)] the induction spectra in Fig. 4 show a voltage minimum followed by a maximum. This characteristic shape originates from the alternating Oersted field that also induces a voltage into the loop. Indeed this signal is subtracted by the reference measurements when there is no vortex. The measured signal is a convolution of the voltage induced by the vortex motion and the voltage induced by the Oersted field. The phase of the signal from the vortex gyration is shifting with respect to the excitation when changing the frequency and leads to a constructive or destructive interference of both contributions. The phase changes by $180^{\circ}$ from frequencies smaller to frequencies larger than the resonance frequency. ${ }^{28}$ This is why there is a formation of a dip of the effective voltage in front of the peak in the induction voltage spectra (see Sec. IV). The pluses in Fig. 5(b) correspond to the differential induction voltages between the minimum and the maximum signal for the single square shown in Fig. 4. The good agreement between the induction signals and the absorption maxima of the ensemble measurements confirms the conclusion that the induction loop senses the magnetization dynamics of the permalloy square. In contrast to the ensemble measurements the inductive measurements show a nonmonotonic behavior of the resonance frequency by changing field strengths continuously. These characteristics originate from irregularities in the inner structure of the permalloy square that locally change the magnetic properties and consequently the resonance frequency. Kamionka et al. observed similar effects on the amplitude of the vortex trajectory. It abruptly changes by varying the excitation energy slightly. ${ }^{29}$

The spectra in Fig. 5(b) exhibit asymmetries in frequency around their resonances. An example is given in Fig. 5(c). The asymmetry could be caused by nonlinear vortex oscillations. When the vortex is treated as a quasiparticle its potential can be assumed to be parabolic ${ }^{28,30}$ if the vortex motion is mainly located in the inner part of the square. Closer to the edge of the square, i.e., for a large vortex gyration radius, nonparabolic terms have to be considered. ${ }^{13,27}$ These terms presumably cause the asymmetric shapes of the resonance curves. Comparison of Fig. 4 and Fig. 5(b) reveal that individual vortex resonances can deviate from averaged measurements. This shows that irregularities such as defects and roughness are important in the description of vortex dynamics.

Defects in Landau-domain patterns can change the resonance frequency of the vortex or pin it. This can be used to read information about the chirality electrically by artificially placing defects on magnetic films. In the induction spectra of Fig. 4 a gap with no sign of magnetization dynamics exists between $-2.0 \mathrm{mT}$ and $0.7 \mathrm{mT}$. In this regime the vortex is presumably pinned close to the center of the square. Figure 6(a) shows a scanning electron micrograph of the investigated square with a contrast that emphasizes defects. If such defects disturb the vortex motion locally the resonance frequency will shift. The defect that is highlighted in Fig. 6(a) is positioned slightly on the right-hand side of the center. Negative (positive) field magnitudes in the $y$ direction shift the vortex to the
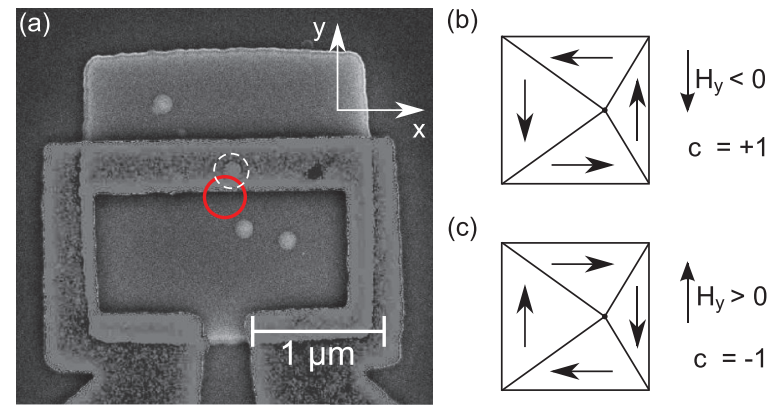

FIG. 6. (Color online) (a) Scanning electron micrograph of the investigated square with an induction loop on top. The dashed line encircles a defect; the solid red circle indicates an undisturbed vortex trajectory. (b) Landau-domain pattern with a chirality of $c=-1$ in a static external field of $H_{y}>0$. (c) Landau-domain pattern with a chirality of $c=+1$ in a static external field of $H_{y}<0$.

right-hand side if its chirality is $+1(-1)$ [see Figs. $6(\mathrm{~b})$ and 6(c)]. The external field interval where the gap occurs lays mainly at negative field values. From the position of the defect in the square and the gap in the spectra shown in Fig. 4 we thus determine a chirality of $c=+1$. Commonly the states $c=+1$ and $c=-1$ are degenerated and at each measurement point in the induction spectra the chirality is not stringently one specific value. But irregularities in the microstructure cause a preferred chirality in the Landau-domain pattern.

\section{DESCRIPTION OF THE INDUCTION SPECTRA}

As described in Sec. III the shape of the induction voltage spectrum has a minimum directly followed by a maximum. This behavior is known around resonances for driven oscillations for the dynamic susceptibility. ${ }^{31}$ The real part of the dynamic susceptibility induces a phase shift of the response of the magnetization to the exciting field whereas its imaginary part leads to Lorentzian-shaped absorption. In the inductive method the superposition of the two voltages, induced by the Oersted field and by the vortex gyration with varying phases, leads to the characteristic shape of the measured spectra. In the following we calculate the effective induction voltage. If it is assumed that the vortex behaves like a quasiparticle in a harmonic potential the phase $\Delta \varphi_{x}\left(\Delta \varphi_{y}\right)$ between the vortex position in the $x$ direction ( $y$ direction) and the exciting Oersted field depends on the frequency of the Oersted field. Figure 7(a) depicts these phases calculated from the equation of vortex motion. ${ }^{28}$ We set a chirality of $c=+1$, a polarization of $p=+1$, and an Oersted field amplitude of $\mu_{0} H_{\mathrm{ext}}=0.21 \mathrm{mT}$. A gyromagnetic ratio of $\gamma=2.21 \times 10^{5} \mathrm{mC}^{-1}$, an edge length of the permalloy square of $l=2.2 \mu \mathrm{m}$, a resonance frequency $\omega /(2 \pi)=178 \mathrm{MHz}$ of the vortex gyration, and a damping in the permalloy of $\Gamma=29 \mathrm{MHz}$ (Ref. 32) is used. To determine the frequency dependence of the effective induction voltage in the loop also the radius of the vortex gyration has to be considered because the trajectory's radius decreases when excited off-resonantly. Figure 7(b) depicts the calculated amplitudes. The amplitudes $A_{x}$ and $A_{y}$ corresponding to the $x$ and $y$ direction are different since the trajectories are elliptical for an arbitrary excitation frequency. 
(a)

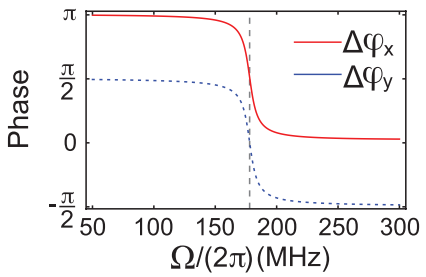

(b)

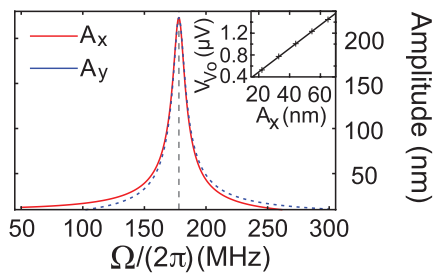

(c)

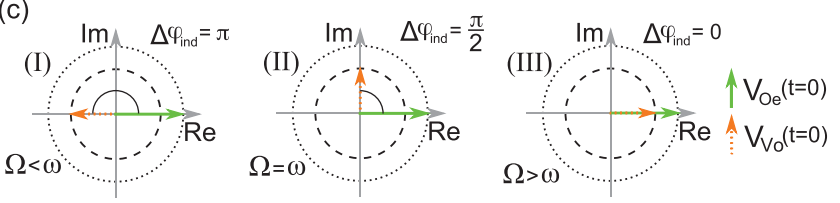

FIG. 7. (Color online) (a) Phases between the vortex displacement in $x$ (red solid) and $y$ (blue dotted) direction and maximal Oersted field versus the excitation frequency $\Omega$ for a square with a lateral size of $2.2 \mu \mathrm{m}$. (b) Amplitude of the vortex gyration versus the excitation frequency. The dashed lines represent the resonance frequency $\omega$ of the vortex. The inset in (b) depicts the dependence of the induction voltage on the amplitude in the $x$ direction simulated for a square with a lateral size of $500 \mathrm{~nm}$ and a thickness of $20 \mathrm{~nm}$. (c) Vector diagrams of the phases between the voltages induced by the Oersted fields from the stripline (green solid arrows) and the vortex gyration (orange dotted arrows) at excitation frequencies $\Omega$ lower than (I), equal to (II), and higher than (III) the resonance frequency $\omega$.

In the experiments it is assumed that the alternating induction voltages from the Oersted field of the stripline with the phase $\varphi_{\mathrm{Oe}}$ and from the vortex gyration with the phase $\varphi_{\mathrm{Vo}}$ induce harmonic signals since both are sinusoidal. In
Fig. 7(c) the phase difference $\Delta \varphi_{\text {ind }}=\varphi_{\mathrm{Vo}}-\varphi_{\mathrm{Oe}}$ at the time $t=0$ between the two flux contributions is shown in vector diagrams for excitation frequencies $\Omega$ lower than, equal to, and larger than the resonance frequency $\omega$ of the vortex. The resonant case in Fig. 7(c) (II) is obtained from the simulations in Fig. 2(b). The negative time derivative of the Oersted field represented by the green solid line is proportional to the induction voltage. Obviously the induction voltage originating from the vortex gyration has a phase difference of $+\pi / 2$ to the induction voltage from the Oersted field. This phase difference depends on the arrangement of the loop as well as on the phases $\Delta \varphi_{x}$ and $\Delta \varphi_{y}$. The simulations in Fig. 2 show that the induction voltage from the vortex gyration is given by the vortex position. By comparing the phases in Figs. 7(a) and 7(c) at resonance it can be concluded that the phase difference $\Delta \varphi_{\text {ind }}$ equals $\Delta \varphi_{x}$. The off-resonant cases in Fig. 7(c) are deduced from Fig. 7(a) by assuming the same frequency-dependent phase shift of $\Delta \varphi_{\text {ind }}(\Omega)$ and $\Delta \varphi_{x}(\Omega)$. Below we assume that the voltage induced by the gyrating vortex is proportional to the vortex displacement justified by the simulations of the induction voltage for different gyration radii shown in the inset of Fig. 7(b). The induced voltage is determined directly above the square with a loop arrangement as depicted in the inset of Fig. 1. For our sample the proportionality constant between the voltage induced by the vortex gyration and the gyration amplitude is about $5.7 \mathrm{Vm}^{-1}$ if a voltage of $1 \mu \mathrm{V}$ and a typical gyration radius of $175 \mathrm{~nm}$ is presumed. For the calculation of the effective induction voltage $V_{\text {eff }}$ the averages of $A_{x}(\Omega)$ and $A_{y}(\Omega)$ are taken as amplitudes to scale the induction voltages.

The effective induction voltage $V_{\text {eff }}$ is given by

$$
V_{\mathrm{eff}}(\Omega)=\lim _{T \rightarrow \infty} \sqrt{\frac{1}{T} \int_{0}^{T}\left\{V_{\mathrm{Oe}} \cos [\Omega t]+V_{\mathrm{Vo}}(\Omega) \cos \left[\Omega t+\Delta \varphi_{\mathrm{ind}}(\Omega)\right]\right\}^{2} d t}
$$

where the amplitude $V_{\mathrm{Oe}}$ describes the voltage induced by the Oersted field from the stripline. The second term with the amplitude $V_{\mathrm{Vo}}(\Omega)$ stands for the induction voltage caused by the gyration of the vortex core. For the geometry of the induction loop used here the phase differences $\Delta \varphi_{\text {ind }}$ and $\Delta \varphi_{x}$ are equal. Thus the voltage

$$
V_{\mathrm{eff}}(\Omega)=\frac{1}{\sqrt{2}} \sqrt{V_{\mathrm{Oe}}^{2}+V_{\mathrm{Vo}}^{2}(\Omega)+2 V_{\mathrm{Oe}} V_{\mathrm{Vo}}(\Omega) \cos \left[\Delta \varphi_{x}(\Omega)\right]}
$$

results. In Fig. 8 a measured induction voltage spectrum at $-4.6 \mathrm{mT}$ (black) is fitted with Eq. (3) (gray) exemplarily. From the fit $200 \mu \mathrm{V}$ have been subtracted to obtain the differential effective induction voltage. This value is set for the voltage $V_{\mathrm{Oe}}$ due to the Oersted field. With the fit we obtain a resonance frequency of $170 \mathrm{MHz}$, a damping constant of $\Gamma=23.2 \mathrm{MHz}$, and an amplitude of the alternating Oersted field of $0.19 \mathrm{mT}$. This amplitude is in good agreement with the amplitude of $0.21 \mathrm{mT}$ calculated with Biot-Savart law. The ratio between the amplitude of the induced voltage due to the vortex motion and the gyration radius is $4.1 \mathrm{Vm}^{-1}$. From the maximum voltage $0.8 \mu \mathrm{V}$ in the measured spectrum of Fig. 8 a radius of $195 \mathrm{~nm}$ is calculated which is in the range of a typical gyration radius. Thus all fit parameters have reasonable values. Further the

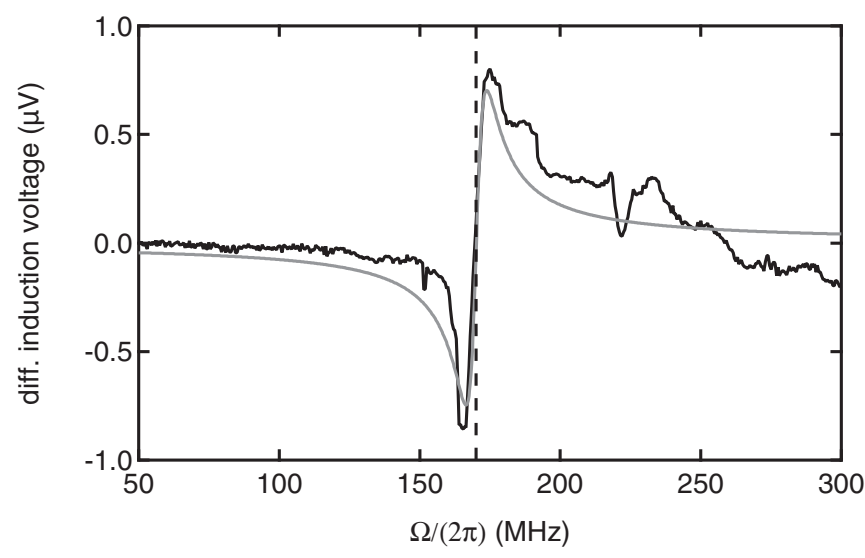

FIG. 8. Differential effective induction voltage versus excitation frequency $\Omega$. The gray curve is a fit with Eq. (3) minus a voltage of $200 \mu \mathrm{V}$ to the experimental curve shown in black measured at $-4.6 \mathrm{mT}$. The dashed line represents the resonance frequency of the vortex $(170 \mathrm{MHz})$. 
shape of the induction voltage spectrum is reproduced by the analytical function and delivers a good description of the measured profile.

\section{CONCLUSION}

We demonstrate the possibility to measure the resonance frequencies of vortex cores in single micrometer-sized permalloy squares with an inductive method. This highly sensitive method allows us to reach a noise level of nanovolts for frequencies in the gigahertz regime. It enables us to sense vortex deflections of a few tens of nanometers. Simulations deliver similar resonance frequencies of the vortex gyration and similar magnitudes of the voltages induced by the magnetization dynamics in the Landau-domain pattern as the experiments. We have shown analytically that the measured induction voltage spectra are composed of voltages induced by the Oersted fields from the stripline and voltages induced due to the gyrating vortex. The chirality of the pattern is determined directly from spectroscopic measurements by analyzing the influence of defects. It is interesting for magnetic memories based on Landau-domain patterns ${ }^{33}$ that the chirality can be read out electrically.

\section{ACKNOWLEDGMENTS}

We thank Ulrich Merkt for fruitful discussions and Michael Volkmann for excellent technical support. Financial support by the Deutsche Forschungsgemeinschaft via Graduiertenkolleg 1286 "Functional Metal-Semiconductor Hybrid Systems" and Sonderforschungsbereich 668 "Magnetism from the single atom to the nanostructure" is gratefully acknowledged. *hlangner@physnet.uni-hamburg.de

${ }^{1}$ A. Hubert and R. Schäfer, Magnetic Domains, 1st ed. (Springer, 1998).

${ }^{2}$ T. J. Silva, C. S. Lee, T. M. Crawford, and C. T. Rogers, J. Appl. Phys. 85, 7849 (1999).

${ }^{3}$ J. Fassbender, J. von Borany, A. Mücklich, K. Potzger, W. Möller, J. McCord, L. Schultz, and R. Mattheis, Phys. Rev. B 73, 184410 (2006).

${ }^{4}$ K. S. Buchanan, M. Grimsditch, F. Y. Fradin, S. D. Bader, and V. Novosad, Phys. Rev. Lett. 99, 267201 (2007).

${ }^{5}$ A. Vogel, A. Drews, T. Kamionka, M. Bolte, and G. Meier, Phys. Rev. Lett. 105, 037201 (2010).

${ }^{6}$ M. Curcic, B. Van Waeyenberge, A. Vansteenkiste, M. Weigand, V. Sackmann, H. Stoll, M. Fähnle, T. Tyliszczak, G. Woltersdorf, C. H. Back, and G. Schütz, Phys. Rev. Lett. 101, 197204 (2008).

${ }^{7}$ R. L. Compton, T. Y. Chen, and P. A. Crowell, Phys. Rev. B 81, 144412 (2010).

${ }^{8}$ K. Perzlmaier, M. Buess, C. H. Back, V. E. Demidov, B. Hillebrands, and S. O. Demokritov, Phys. Rev. Lett. 94, 057202 (2005).

${ }^{9}$ W. K. Hiebert, A. Stankiewicz, and M. R. Freeman, Phys. Rev. Lett. 79, 1134 (1997).

${ }^{10}$ T. Y. Chen, A. T. Galkiewicz, and P. A. Crowell, Phys. Rev. B 85, 180406 (2012).

${ }^{11}$ B. Van Waeyenberge, A. Puzic, H. Stoll, K. W. Chou, T. Tyliszczak, R. Hertel, M. Fähnle, H. Brückl, K. Rott, G. Reiss, I. Neudecker, D. Weiss, C. H. Back, and G. Schütz, Nature (London) 444, 461 (2006).

${ }^{12}$ M. Bolte, G. Meier, B. Krüger, A. Drews, R. Eiselt, L. Bocklage, S. Bohlens, T. Tyliszczak, A. Vansteenkiste, B. Van Waeyenberge, K. W. Chou, A. Puzic, and H. Stoll, Phys. Rev. Lett. 100, 176601 (2008).

${ }^{13}$ H. H. Langner, T. Kamionka, M. Martens, M. Weigand, C. F. Adolff, U. Merkt, and G. Meier, Phys. Rev. B 85, 174436 (2012).

${ }^{14}$ S.-B. Choe, Y. Acremann, A. Scholl, A. Bauer, A. Doran, J. Stöhr, and H. A. Padmore, Science 304, 420 (2004).

${ }^{15}$ J. Raabe, C. Quitmann, C. H. Back, F. Nolting, S. Johnson, and C. Buehler, Phys. Rev. Lett. 94, 217204 (2005).
${ }^{16}$ C. M. Schneider, A. Kuksov, A. Krasyuk, A. Oelsner, D. Neeb, S. A. Nepijko, G. Schönhense, I. Mönch, R. Kaltofen, J. Morais, C. de Nadaï, and N. B. Brookes, Appl. Phys. Lett. 85, 2562 (2004).

${ }^{17}$ J. Miguel, J. Sánchez-Barriga, D. Bayer, J. Kurde, B. Heitkamp, M. Piantek, F. Kronast, M. Aeschlimann, H. A. Dürr, and W. Kuch, J. Phys.: Condens. Matter 21, 496001 (2009).

${ }^{18}$ K. Nakano, D. Chiba, K. Sekiguchi, S. Kasai, N. Ohshima, K. Kobayashi, and T. Ono, Appl. Phys. Expr. 3, 053001 (2010).

${ }^{19}$ M. Hayashi, S. Kasai, and S. Mitani, Appl. Phys. Expr. 3, 113004 (2010).

${ }^{20}$ M. Hayashi, J. Ieda, Y. Yamane, J. I. Ohe, Y. K. Takahashi, S. Mitani, and S. Maekawa, Phys. Rev. Lett. 108, 147202 (2012).

${ }^{21}$ A. Drews, B. Krüger, G. Meier, S. Bohlens, L. Bocklage, T. Matsuyama, and M. Bolte, Appl. Phys. Lett. 94, 062504 (2009).

${ }^{22}$ K. W. Chou, A. Puzic, H. Stoll, D. Dolgos, G. Schütz, B. Van Waeyenberge, A. Vansteenkiste, T. Tyliszczak, G. Woltersdorf, and C. H. Back, Appl. Phys. Lett. 90, 202505 (2007).

${ }^{23}$ L. Breitenstein, P. Lendecke, S. Bohlens, G. Meier, and U. Merkt, J. Appl. Phys. 104, 083909 (2008).

${ }^{24}$ A. Wachowiak, J. Wiebe, M. Bode, O. Pietzsch, M. Morgenstern, and R. Wiesendanger, Science 298, 577 (2002).

${ }^{25}$ M. J. Donahue and D. G. Porter, OOMMF User's Guide, Version 1.0, Interagency Report NIST IR 6376, Gaithersburg, MD, 1999.

${ }^{26}$ K. S. Buchanan, P. E. Roy, M. Grimsditch, F. Y. Fradin, K. Y. Guslienko, S. D. Bader, and V. Novosad, Phys. Rev. B 74, 064404 (2006).

${ }^{27}$ A. Drews, B. Krüger, G. Selke, T. Kamionka, A. Vogel, M. Martens, U. Merkt, D. Möller, and G. Meier, Phys. Rev. B 85, 144417 (2012).

${ }^{28}$ B. Krüger, A. Drews, M. Bolte, U. Merkt, D. Pfannkuche, and G. Meier, Phys. Rev. B 76, 224426 (2007).

${ }^{29}$ T. Kamionka, M. Martens, K. W. Chou, A. Drews, T. Tyliszczak, H. Stoll, B. Van Waeyenberge, and G. Meier, Phys. Rev. B 83, 224422 (2011)

${ }^{30}$ A. A. Thiele, Phys. Rev. Lett. 30, 230 (1973).

${ }^{31}$ D. D. Stancil and A. Prabhakar, Spin Waves-Theory and Applications, 1st ed. (Springer, 2009).

${ }^{32}$ The damping $\Gamma$ is estimated from Eq. (14) in Ref. 28.

${ }^{33}$ S. Bohlens, B. Krüger, A. Drews, M. Bolte, G. Meier, and D. Pfannkuche, Appl. Phys. Lett. 93, 142508 (2008). 